deviations from HWE revealed no significant departures $(P>0.05)$ at eight loci; conversely, a significant excess of homozygotes was observed at one locus (ST2-41). The use of MICRO-CHECKER software (Van Oosterhout et al. 2004) indicated that the significant deviations from HWE at this locus may be the result of null alleles, although no scoring errors were found associated with stuttering bands or large allele dropout. ARLEQUIN was also used to test for gametic disequilibrium by application of a Bonferroni correction for multiple comparisons (Rice 1989). No evidence of gametic disequilibrium was detected for any pairs of loci.

The relatively small number of useful loci for $S$. terebrans, resulting from an initially promising set of positive clones, suggests that the isolation of suitable microsatellite loci for this group of Crustaceans is not easy, as also demonstrated by the small number of simple sequence repeats isolated in isopods thus far (Grandjean et al. 2005; Verne et al. 2006; Haye \& Marchant 2007, 2008; Leese et al. 2008). Nevertheless, we are confident that the loci characterized in this study are suitable to infer parentage, relatedness and phylogeographic patterns in this taxon, which probably includes some cryptic species.

\section{Acknowledgements}

We thank Sara Fratini and Rocco Rorandelli. We also thank Eleonora Trajano for the help rendered in collecting material. The research was funded by the Italian Ministry of University and Research (MIUR) (FIRB/2001).

\section{References}

Baratti M, Goti E, Messana G (2005) High level of genetic differentiation in the marine isopod Sphaeroma terebrans (Crustacea Isopoda Sphaeromatidae) as inferred by mitocondrial DNA analysis. Journal of Experimental Marine Biology and Ecology, 315, 225-234.
Excoffier L, Laval G, Schneider S (2005) ARLEQUIN ver. 3.0: an integrated software package for population genetics data analysis. Evolutionary Bioinformatics Online, 1, 47-50.

Goudet J (2001) FSTAT: A program to estimate and test gene diversities and fixation indices (v. 2.9.3). Available from http://www2. unil.ch/popgen/softwares/fstat.htm.

Grandjean F, Gouin N, Verne S, Delaunay C, Patri S (2005) Characterization of polymorphic microsatellite loci in the terrestrial isopod Porcellionides pruinosus. Molecular Ecology Notes, 5, 503-507.

Haye PA, Marchant S (2007) Newly developed PCR primers for polymorphic microsatellite loci from the marine isopod Limnoria sp. Molecular Ecology Notes, 7, 1245-1247.

Haye PA, Marchant S (2008) Isolation and characterization of 11 polymerase chain reaction primers for microsatellite loci for the Chilean marine isopod Excirolana hirsuticauda. Molecular Ecology Resources, 8, 1088-1090.

Leese F, Kop A, Agrawal S, Held C (2008) Isolation and characterization of microsatellite markers from the marine isopods Serolis paradoxa and Septemserolis septemcarinata (Crustacea: Peracarida). Molecular Ecology Resources, 8, 818-821.

Rice WR (1989) Analyzing tables of statistical tests. Evolution, 43, 223-225.

Rozen S, Skaletsky H (2000) Primer 3 on the WWW for general users and for biologist programmers. Methods in Molecular Biology, 132, 365-386.

Van Oosterhout C, Hutchinson WF, Wills DPM, Shipley P (2004) MICRO-CHECKER: software for identifying and correcting genotyping errors in microsatellite data. Molecular Ecology Notes, 4, 535-538.

Verne S, Puillandre N, Brunet G et al. (2006) Characterization of polymorphic microsatellite loci in the terrestrial isopod Armadillidium vulgare. Molecular Ecology Notes, 6, 328-330.

Zane L, Bargelloni L, Patarnello T (2002) Strategies for microsatellites isolation: a review. Molecular Ecology, 11, 1-16.

doi: $10.1111 / j .1755-0998.2009 .02666 . x$

(C) 2009 Blackwell Publishing Ltd

\title{
Characterization of Mauritius parakeet (Psittacula eques) microsatellite loci and their cross-utility in other parrots (Psittacidae, Aves)
}

\author{
CLAIRE RAISIN,$*+£$ DEBORAH A. DAWSON,$*$ ANDREW G. GREENWOOD, CARL G. JONESI§ and \\ JIM J. GROOMBRIDGE† \\ *NERC Molecular Genetics Facility, Department of Animal and Plant Sciences, University of Sheffield, Sheffield S10 2TN, UK, \\ †Durrell Institute of Conservation \& Ecology, University of Kent, Canterbury CT2 7NZ, UK, †International Zoo Veterinary Group, \\ Keighley Business Centre, South Street, Keighley, W. Yorkshire BD21 1AG, UK, §Mauritian Wildlife Foundation, Grannum Road, \\ Vacoas, Mauritius, IIDurrell Wildlife Conservation Trust, Les Augres Manor, Trinity, Jersey JE3 5BP, UK
}

\begin{abstract}
We characterized 21 polymorphic microsatellite loci in the endangered Mauritius parakeet (Psittacula eques). Loci were isolated from a Mauritius parakeet genomic library that had been enriched separately for eight different repeat motifs. Loci were characterized in up to
\end{abstract}




\begin{abstract}
43 putatively unrelated Mauritius parakeets from a single population inhabiting the Black River Gorges National Park, Mauritius. Each locus displayed between three and nine alleles, with the observed heterozygosity ranging between 0.39 and 0.96 . All loci were tested in 10 other parrot species. Despite testing few individuals, between seven and 21 loci were polymorphic in each of seven species tested.
\end{abstract}

Keywords: AVES, cross-species utility, Mauritius parakeet, microsatellite, parrot, Psittacidae,

Received 22 December 2008; accepted 3 February 2009

The Mauritius parakeet (Psittacula eques) is the only endemic psittacine remaining in the Mascarene Islands and is classified as endangered (IUCN 2008). The population declined to less than 20 individuals (including only three known breeding females) in the early 1980s but currently numbers approximately 360, all of which are found in the Black River Gorges National Park, South West Mauritius (Malham et al. 2006). We have characterized a set of polymorphic microsatellite loci for the Mauritius parakeet.

Blood was collected from 43 Mauritius parakeet individuals and stored in 70-90\% ethanol at room temperature. For library construction, genomic DNA was extracted from blood using a DNeasy Blood and Tissue Kit (QIAGEN Ltd). Mauritius parakeet microsatellites were isolated from an enriched genomic library prepared essentially as Jones et al. (2002) by Genetic Identification Services. The pooled genomic DNA of three Mauritius parakeet individuals (one male and two females) was digested with blunt ended-restriction enzymes (AluI, DraI, FspI, HpaI, MscI, NaeI). Fragments (350-700 bp) were ligated to the double-stranded linker sequence $5^{\prime}$ AAGCTTTCGTTTTACAACGTCGTGG-3' (which contains a HindIII site at the $5^{\prime}$ end) and hybridized separately against one of eight target repeat sequences: $(\mathrm{CA})_{15},(\mathrm{GA})_{15},(\mathrm{AAT})_{15}$, $(\mathrm{ATG})_{12},(\mathrm{CAG})_{10},(\mathrm{CATC})_{8},(\mathrm{TAGA})_{8}$ and $(\mathrm{TGAC})_{8}$. The enriched fragments were digested at the HindIII site of the linker and then ligated into pUC19-HindIII. Colonies were not screened for repeat motifs but directly sequenced. A total of 104 clones were sequenced in the forward orientation using an M13-based forward primer (5'-AGGAAACAGC TATGACCATG-3'). Primer sets were designed for 48 loci using DesignerPCR version 1.03 (Research Genetics, Inc.) and used to amplify eight unrelated Mauritius parakeet individuals to assess polymorphism. When initially amplified in eight unrelated individuals and screened on agarose gel, 35 loci appeared polymorphic, eight monomorphic and five failed to amplify or were unscorable (unpublished data). Twenty-one polymorphic loci (enriched for $(\mathrm{CA})_{15}$ $(\mathrm{GA})_{15},(\mathrm{CATC})_{8}$ and (TAGA) $)_{8}$ motifs) were selected for further optimization.

Genomic DNA was extracted from blood using ammonium acetate following the method used by Nicholls et al. (2000) and used for PCR. A range of annealing temperatures $\left(50^{\circ} \mathrm{C}-\right.$ $68^{\circ} \mathrm{C}$ ) were tested and the temperature producing the cleanest and strongest PCR product when observed on an $0.8 \%$ agarose gel stained with ethidium bromide was selected for PCR.
Each locus was amplified separately. Each $2 \mu \mathrm{L}$ PCR contained approximately $10 \mathrm{ng}$ of genomic DNA with $0.2 \mu \mathrm{M}$ of each primer and $1 \mu \mathrm{L}$ QIAGEN multiplex PCR mix (QIAGEN Inc.). PCR amplification was performed using a DNA Engine Tetrad 2 thermal cycler (MJ Research, Bio-Rad). The PCR profile used was $95^{\circ} \mathrm{C}$ for $15 \mathrm{~min}$; then 40 cycles of $94{ }^{\circ} \mathrm{C}$ for $30 \mathrm{~s}, \mathrm{~T}_{\mathrm{a}}{ }^{\circ} \mathrm{C}$ (Table 1 ) for $90 \mathrm{~s}, 72{ }^{\circ} \mathrm{C}$ for $90 \mathrm{~s}$, followed by one cycle of $10 \mathrm{~min}$ at $72{ }^{\circ} \mathrm{C}$. Genotypes were scored on an ABI 3730 DNA Analyser with GeneScan ROX-500 size standard using GeneMapper software (Applied Biosystems). Each locus was loaded on the ABI 3730 separately. Multiplexing was not performed pre- or post-PCR amplification.

All 21 loci were polymorphic and the number of alleles observed when tested in 24-43 individuals from the recovered population ranged from three to nine (Table 1). The DNA sequences of the 21 polymorphic loci were confirmed to be free of vector and unique using BLASTN version 2.2.4 (Altschul et al. 1997). Loci were checked for sex linkage by genotyping individuals of known sex (15 females and 14 males; sex based on morphology). Two loci (Peq16 and Peq21) were found to be Z-linked, therefore only males were characterized at these loci as females were always homozygous.

Observed and expected heterozygosities, and null allele frequencies were calculated using Cervus version 2.0 (Marshall et al. 1998). Tests for departures from Hardy-Weinberg and linkage equilibrium were conducted using a Markov chain method implemented in GenePop version 3.4 (Raymond \& Rousset 1995). One locus (Peq08) deviated from Hardy-Weinberg equilibrium and also displayed a high predicted null allele frequency (Table 1).

Based on sequence homology, 15 of the 21 loci could be assigned a chromosome location in the zebra finch (Taeniopygia guttata) genome assembly (compiled by the Washington University School of Medicine Genome Sequencing Centre, http://genome.wustl.edu/genome.cgi?GENOME=Taenio pygia\%20guttata\&SECTION=assemblies) and 11 loci were assigned a chicken (Gallus gallus) chromosome location (using a WU-BLAST with distant homologies settings implemented on the ENSEMBL webpage www.ensembl.org/ Gallus_gallus/index.html; see Dawson et al. 2006; Table 1, Fig. 1). Loci Peq16 and Peq21 were assigned to the zebra finch $Z$ chromosome (Table 1, Fig. 1).

Fifteen known family groups (both parents and at least one offspring) were genotyped and used to test for linkage 
Table 1 Characterization of Mauritius parakeet Psittacula eques microsatellite loci (Psittacidae, Aves)

\begin{tabular}{|c|c|c|c|c|c|c|c|c|c|c|c|c|c|c|c|c|}
\hline Locus & $\begin{array}{l}\text { EMBL } \\
\text { accession } \\
\text { number }\end{array}$ & $\begin{array}{l}\text { Chr. location } \\
\text { (see footnote } \\
\text { and text) }\end{array}$ & $\begin{array}{l}\text { Chr. } \\
\text { location } \\
\text { (bp) }\end{array}$ & $\begin{array}{l}\text { BLA } \\
\text { ST } \\
\text { P(N) }\end{array}$ & Repeat motif & $\begin{array}{l}\text { Fluoro-label } \\
\text { (F) }\end{array}$ & Primer sequences $\left(5^{\prime}-3^{\prime}\right)$ & $\begin{array}{l}\text { Optimised } \\
T_{a}\left({ }^{\circ} \mathrm{C}\right)\end{array}$ & $n$ & $A$ & $\begin{array}{l}\text { Exp. } \\
\text { allele } \\
\text { size (bp)‡ }\end{array}$ & $\begin{array}{l}\text { Obs. } \\
\text { allele } \\
\text { sizes (bp) }\end{array}$ & $H_{\mathrm{O}}$ & $H_{\mathrm{E}}$ & $P_{\mathrm{HWE}}$ & $\begin{array}{l}\text { Est. null } \\
\text { allele } \\
\text { freq. }\end{array}$ \\
\hline Peq01 & FM865414 & Tgu2 & 61550289 & $3 \mathrm{E}-28$ & $(\mathrm{CA})_{13}$ & HEX & $\begin{array}{l}\text { F: AGGCTTAACAGATGTAGGACAC } \\
\text { R: TGTGCTTTTCCATCACAAG }\end{array}$ & 63 & 42 & 6 & 202 & $189-209$ & 0.67 & 0.58 & 0.87 & -0.16 \\
\hline Peq02 & FM865415 & No strong hits & & & $(\mathrm{GATG})_{10}$ & 6-FAM & $\begin{array}{l}\text { F: CTTTGAGCACCCACACAAC } \\
\text { R: GTGTCGCAACCTCCTTATG }\end{array}$ & 66 & 43 & 3 & 145 & $144-153$ & 0.47 & 0.53 & 0.77 & 0.04 \\
\hline Peq03 & FM865416 & Tgu1 & 79810332 & $4 \mathrm{E}-18$ & $\begin{array}{l}(\mathrm{TAGA})_{12}(\mathrm{CAGA})_{2} \\
(\mathrm{TAGA})_{1}(\mathrm{CAGA})_{1}\end{array}$ & 6-FAM & $\begin{array}{l}\text { F: GGATGTTCAGTTCTCTTGTTTC } \\
\text { R: ATGGTTCTCCTGTGTCTATCAG }\end{array}$ & 66 & 42 & 7 & 287 & $274-300$ & 0.76 & 0.76 & 0.20 & 0.09 \\
\hline Peq04 & FM865417 & Tgu4 & 40782899 & $2 \mathrm{E}-38$ & $(\mathrm{TAGG})_{3}(\mathrm{TAGA})_{10}$ & HEX & $\begin{array}{l}\text { F: GGATTTCTCATGCCTAAAATG } \\
\text { R: TGACAAGTCCACCAAAAATATC }\end{array}$ & 56 & 43 & 5 & 287 & $285-306$ & 0.65 & 0.63 & 0.37 & 0.04 \\
\hline Peq05 & FM865418 & Tgu1 & 39968225 & $1 \mathrm{E}-41$ & $(\mathrm{CA})_{11}$ & 6-FAM & $\begin{array}{l}\text { F: GGAATTGTAGGTTTTAATGCAC } \\
\text { R: AGCTCATAAACAGCCATATCTC }\end{array}$ & 56 & 42 & 4 & 133 & $125-131$ & 0.57 & 0.60 & 0.52 & -0.01 \\
\hline Peq06 & FM865419 & Tgu4 & 43705460 & 7E-42 & $(\mathrm{TG})_{13}$ & HEX & $\begin{array}{l}\text { F: CAAAAGAGCATCAATGGTATG } \\
\text { R: GCTAACACCCCCCTATCC }\end{array}$ & 59 & 41 & 7 & 216 & 213-244 & 0.83 & 0.79 & 0.89 & -0.03 \\
\hline Peq07 & FM865420 & Tgu4 & 59562728 & $4 \mathrm{E}-13$ & $(\mathrm{CA})_{11}$ & HEX & $\begin{array}{l}\text { F: AAACAAACATACCCACAGAAAC } \\
\text { R: GGAGGATAAGCAGAACTTGAG }\end{array}$ & 66 & 42 & 3 & 128 & $114-124$ & 0.40 & 0.34 & 0.64 & -0.06 \\
\hline Peq08+ & FM865421 & Gga27 & 2668172 & $9 \mathrm{E}-15$ & $(\mathrm{GATG})_{9}$ & 6-FAM & $\begin{array}{l}\text { F: AGTCGGGAACAGTTTCATTAG } \\
\text { R: GACATGATGCTGACACAGATAG }\end{array}$ & 58 & 42 & 9 & 255 & 235-265II & 0.48 & 0.68 & 0.00 & 0.12 \\
\hline Peq09 & FM865422 & Tgu2 & 154849943 & $1 \mathrm{E}-24$ & $\begin{array}{l}(\mathrm{CATC})_{10} \mathrm{CACC} \\
(\mathrm{CATC})_{6}\end{array}$ & HEX & $\begin{array}{l}\text { F: GGTGGGTTGTGTGAAAGAA } \\
\text { R: GGACTGTGGATGGAGAGGT }\end{array}$ & 56 & 41 & 6 & 218 & $206-242$ & 0.81 & 0.80 & 0.78 & 0.04 \\
\hline Peq10 & FM865423 & Tgu3 & 17147094 & $2 \mathrm{E}-48$ & $(\mathrm{TAGA})_{14}$ & 6-FAM & $\begin{array}{l}\text { F: ACCCTTCCAAGAGAGTTTTAAG } \\
\text { R: GGATCTTTCCTTTATCTGTGTG }\end{array}$ & 59 & 42 & 6 & 133 & $109-129$ & 0.76 & 0.80 & 0.35 & 0.01 \\
\hline Peq11 & FM865424 & Tgu3 & 30982572 & $4 \mathrm{E}-31$ & $\begin{array}{l}(\mathrm{TCTA})_{14} \text { TCTTA } \\
(\mathrm{TCTA})_{2}\end{array}$ & 6-FAM & $\begin{array}{l}\text { F: CTCAAGGAGAATCTGAAGTCTG } \\
\text { R: TGGGAGGTTAGAGTGAAAAAC }\end{array}$ & 56 & 42 & 8 & 282 & $253-281 €$ & 0.81 & 0.81 & 0.64 & 0.06 \\
\hline Peq12 & FM865425 & Tgu2§ & 154288876 & $1 \mathrm{E}-33$ & $(\mathrm{TAGA})_{11}$ & HEX & $\begin{array}{l}\text { F: CACTCGGGATTCTGTGTTAG } \\
\text { R: CCTTAGTCATCGTGCTTGTAG }\end{array}$ & 59 & 41 & 6 & 279 & 271-291 & 0.90 & 0.82 & 0.95 & 0.00 \\
\hline Peq13 & FM865426 & No strong hits & & & $(\mathrm{TG})_{8} \mathrm{~T}(\mathrm{TG})_{7}$ & HEX & $\begin{array}{l}\text { F: GGAACATTCCAAAGCTCAAAGT } \\
\text { R: TTGCTGCACTGTAGTTCTGTGA }\end{array}$ & 55 & 24 & 4 & 125 & 114-122 & 0.67 & 0.65 & 0.97 & -0.04 \\
\hline Peq14 & FM865427 & None & & & $(\mathrm{CA})_{12}$ & NED & $\begin{array}{l}\text { F: GGCTACCATCCTATTTGAAAGA } \\
\text { R: ACGCTGGATCATAACAGAGTAA }\end{array}$ & 58 & 23 & 4 & 219 & $209-225$ & 0.39 & 0.34 & 1.00 & -0.10 \\
\hline Peq15 & FM865428 & Tgu12 & 6379632 & $6 \mathrm{E}-41$ & $(\mathrm{CA})_{14}$ & NED & $\begin{array}{l}\text { F: CTTCATTTTCCTGTGCAGTCTA } \\
\text { R: GGAGTTTTTTGTCGTTTTCTGT }\end{array}$ & 59 & 23 & 9 & 206 & $205-221 €$ & 0.96 & 0.86 & 0.31 & -0.07 \\
\hline Peq16 & FM865429 & TguZ & 43664061 & $1 \mathrm{E}-16$ & $(\mathrm{GATG})_{8}$ & 6-FAM & $\begin{array}{l}\text { F: GCTCCTAAGCCTTTGTAAAGAC } \\
\text { R: CATGTTTGCTTGTCCAGTTTAT }\end{array}$ & 63 & $\begin{array}{l}14 \\
\text { (males) }\end{array}$ & 3 & 136 & $131-149$ & 0.50 & 0.43 & 1.00 & - \\
\hline Peq17 & FM865430 & No strong hits & & & $(\mathrm{CATC})_{13}$ & HEX & $\begin{array}{l}\text { F: ACCTCACAGACACAAAAGGATA } \\
\text { R: GAAATGATAGGAACAGACAGT }\end{array}$ & 62 & 22 & 7 & 228 & $196-224$ & 0.85 & 0.78 & 0.60 & -0.07 \\
\hline Peq18 & FM865431 & Gga10 & 641761 & 7E-19 & $\begin{array}{l}(\mathrm{TAGA})_{2} \mathrm{~T}(\mathrm{TAGA})_{1} \\
\operatorname{TAA}(\mathrm{TAGA})_{3}\end{array}$ & HEX & $\begin{array}{l}\text { F: AGCAAGTAGATTTGGTCGTG } \\
\text { R: TTCTCCCAGAGCACACAC }\end{array}$ & 59 & 24 & 6 & 170 & 155-179 & 0.78 & 0.73 & 0.92 & 0.00 \\
\hline Peq19 & FM865432 & Tgu1A & 22948103 & $3 \mathrm{E}-15$ & $\begin{array}{l}(\mathrm{TCTA})_{7} \mathrm{TC}(\mathrm{CATC})_{10} \\
\operatorname{TATC}(\mathrm{CATC})_{1}\end{array}$ & 6-FAM & $\begin{array}{l}\text { F: GAGCCTCCTACCATAGTTGC } \\
\text { R: CTTCAAATTCAGCAAACTTGTC }\end{array}$ & 58 & 23 & 5 & 230 & $202-234$ & 0.87 & 0.79 & 0.68 & -0.07 \\
\hline Peq20 & FM865433 & Tgu20 & 2718287 & $1 \mathrm{E}-08$ & $\begin{array}{l}(\text { TCTA })_{6} \&(\text { ATCA })_{4} \\
\text { ATCTA }(\text { TCTA })_{5}\end{array}$ & 6-FAM & $\begin{array}{l}\text { F: GATGCAAATGTTACAGCAGTGT } \\
\text { R: AACCCAATCTGTCTGAGAACAG }\end{array}$ & 61 & 23 & 4 & 235 & $223-235$ & 0.74 & 0.71 & 0.51 & -0.04 \\
\hline Peq21 & FM865434 & TguZ & 612947 & 9E-13 & $\begin{array}{l}\left.(\text { TAGA })_{3} \text { T(TAGA }\right)_{2} \\
\text { TTGA }(\text { TAGA })_{1} \mathrm{~T} \\
(\mathrm{TAGA})_{1} \text { TAGG } \\
(\mathrm{TAGA})_{10}\end{array}$ & 6-FAM & $\begin{array}{l}\text { F: CCCAGAGTGGTAGAGAAAGAT } \\
\text { R: GCTGTGTTCAAAAGGGTATG }\end{array}$ & 59 & $\begin{array}{l}14 \\
\text { (males) }\end{array}$ & 5 & 180 & 159-179 & 0.56 & 0.55 & 0.72 & - \\
\hline
\end{tabular}

Chromosome location: more loci could be assigned a location in the zebra finch genome than the chicken genome (15 vs. 11) and no chromosome assignments conflicted; therefore, when the same chromosome was assigned in both genomes only the location in zebra finch is presented. Five loci were assigned a location of the zebra finch genome only (Peq01, Peq03, Peq07, Peq16 and Peq20) and on two occasions, a location could only be assigned in the chicken genome (Peq08 and Peq18). Tgu, zebra finch chromosome; Gga, chicken chromosome. Peq16 and Peq21 are Z-linked loci and therefore were characterized in male individuals only. tDeviation from Hardy-Weinberg equilibrium as identified by GenePop version 3.4 (Raymond \& Rousset 1995; $P<0.001$ ). $T_{a}$ a annealing temperature; $n$, number of unrelated Mauritius parakeet individuals genotyped from the population inhabiting Black River Gorges National Park, South West Mauritius. $A$, number of alleles; fexpected allele size based on length of the Mauritius parakeet sequenced clone; $H_{\mathrm{O}}$, observed heterozygosity; $H_{\mathrm{E}}$, expected heterozygosity; $P_{\mathrm{HWE}}$, Hardy-Weinberg equilibrium test $P$ value as identified by GenePop version 3.4 ; $\$$ multiple other weaker hits also detected; Isome observed allele sizes increased by only $1 \mathrm{bp}$ and may be difficult to score. 
Table 2 Allele sizes observed in other species when amplified using Mauritius parakeet (Psittacula eques) microsatellite loci*

\begin{tabular}{|c|c|c|c|c|c|c|c|c|c|c|c|c|c|c|}
\hline & $\begin{array}{l}\text { Ringneck } \\
\text { parakeet } \\
\text { (Rose- } \\
\text { ringed) }\end{array}$ & $\begin{array}{l}\text { Longtailed } \\
\text { parakeet }\end{array}$ & $\begin{array}{l}\text { Derbyan } \\
\text { parakeet }\end{array}$ & $\begin{array}{l}\text { Moustached } \\
\text { parakeet }\end{array}$ & $\begin{array}{l}\text { Blossom } \\
\text { parakeet }\end{array}$ & $\begin{array}{l}\text { Slaty-headed } \\
\text { parakeet }\end{array}$ & $\begin{array}{l}\text { Alexandrine } \\
\text { parakeet }\end{array}$ & $\begin{array}{l}\text { Eclectus } \\
\text { parrot }\end{array}$ & $\begin{array}{l}\text { Black-lored } \\
\text { parrot }\end{array}$ & $\begin{array}{l}\text { Vasa } \\
\text { parrot }\end{array}$ & $\begin{array}{l}\text { Number of } \\
\text { parrot } \\
\text { species } \\
\text { polymorphic }\end{array}$ & Blue crane & Chicken & Crocodile \\
\hline & $\begin{array}{l}\text { Psittacula } \\
\text { krameri }\end{array}$ & $\begin{array}{l}\text { Psittacula } \\
\text { longicauda }\end{array}$ & $\begin{array}{l}\text { Psittacula } \\
\text { derbiana }\end{array}$ & $\begin{array}{l}\text { Psittacula } \\
\text { alexandri }\end{array}$ & $\begin{array}{l}\text { Psittacula } \\
\text { roseata }\end{array}$ & $\begin{array}{l}\text { Psittacula } \\
\text { himalayana } \\
\text { himalayana }\end{array}$ & $\begin{array}{l}\text { Psittacula } \\
\text { eupatria }\end{array}$ & $\begin{array}{l}\text { Eclectus } \\
\text { roratus } \\
\text { roratus }\end{array}$ & $\begin{array}{l}\text { Tanygnathus } \\
\text { gramineus }\end{array}$ & $\begin{array}{l}\text { Coracopsis } \\
\text { vasa }\end{array}$ & & $\begin{array}{l}\text { Anthropoides } \\
\text { paradisea }\end{array}$ & $\begin{array}{l}\text { Gallus } \\
\text { gallus }\end{array}$ & $\begin{array}{l}\text { Crocodylus } \\
\text { porosus }\end{array}$ \\
\hline Locus & $\begin{array}{l}n=10 \\
\text { (number } \\
\text { of alleles) }\end{array}$ & $n=1$ & $n=1$ & $n=2$ & $n=1$ & $n=1$ & $n=2$ & $n=2$ & $n=1$ & $n=2$ & & $n=1$ & $n=1$ & $n=1$ \\
\hline Peq01 & 189-203 (7) & $x$ & 199,203 & $184,195,210$ & 195,199 & 197 & 195,199 & $193,205,209$ & 195,203 & 194 & 7 & $x$ & 195 & $x$ \\
\hline Peq02 & 144-190 (4) & 149,157 & $x$ & $x$ & $x$ & $x$ & $x$ & $x$ & $x$ & $x$ & 2 & $x$ & $x$ & $x$ \\
\hline Peq03 & $282-306(7)$ & $x$ & $x$ & $x$ & $x$ & $x$ & $x$ & $x$ & $x$ & $x$ & 0 & $x$ & $x$ & $x$ \\
\hline Peq04 & 297,317 (2) & 194,219 & 190 & 211,219 & 213,223 & $192,202,282$ & 189,213 & $104,108^{\S}$ & 208,219 & 192 & 8 & 176 & 295 & 173 \\
\hline Peq05 & $121-125$ (3) & 123 & 120 & 116 & 124,126 & 122 & 123,125 & 136,138 & 120 & 120,131 & 5 & 131 & $x$ & $x$ \\
\hline Peq06 & $217-238$ (5) & 203 & 203 & 200 & $x$ & 236 & 234,238 & 201 & 203 & $x$ & 2 & $x$ & $x$ & $x$ \\
\hline Peq07 & $122-126$ (3) & 112 & $x$ & 116,128 & 110 & $x$ & $x$ & 168,172 & $x$ & 173,175 & 4 & $x$ & $x$ & $x$ \\
\hline Peq08 & 251-259 (3) & 272,277 & 276,280 & 263,288 & 251,259 & $x$ & 251,255 & $259,279,291$ & 235 & 255,263 & 8 & $x$ & $x$ & $x$ \\
\hline Peq09 & $195-235$ (6) & 242,246 & $x$ & $257,261,265$ & 234,258 & $x$ & 191,222 & 175 & $x$ & $x$ & 5 & $x$ & $x$ & $x$ \\
\hline Peq10 & $117-129$ (4) & $x$ & 109,125 & 132,136 & $x$ & 113,129 & 121,125 & 100 & 113,129 & 113 & 6 & 113,129 & $x$ & 113,129 \\
\hline Peq11 & $241-273(7)$ & $308,343^{\S}$ & 264 & $239,243,267,270$ & 268 & 264 & $249,256,260,264$ & 279,287 & 272,276 & $240,248,252$ & 7 & $x$ & $x$ & $x$ \\
\hline Peq12 & 275-295 (4) & 243,332 & 283,337 & 322,348 & $x$ & $x$ & 291,299 & 234,270 & 284,308 & $x$ & 7 & $x$ & $x$ & $x$ \\
\hline Peq13 & $108-123(7)$ & 109 & 114,116 & 116 & $x$ & 104,127 & 108 & 104,108 & 108 & 100 & 4 & 116,122 & 116,123 & 123 \\
\hline Peq14 & 222,226 (2) & $x$ & $\mathrm{x}$ & $x$ & $x$ & $\mathrm{x}$ & $\mathrm{x}$ & $\mathrm{x}$ & $x$ & $x$ & 1 & $x$ & $\mathrm{x}$ & $x$ \\
\hline Peq15 & $188-217$ (3) & $x$ & $x$ & $x$ & $x$ & $x$ & $x$ & $x$ & $x$ & $x$ & 1 & $x$ & $x$ & $x$ \\
\hline Peq16 & 135-144 (3) & 134 & 135 & 131,135 & 161 & $210^{\S}$ & 144,153 & $131,140,144$ & 126 & $x$ & 4 & $x$ & $x$ & $x$ \\
\hline Peq17 & $189-223(6)$ & 233,241 & 201,205 & $201,205,208$ & 234 & $x$ & 217,221 & 205,209 & 213,217 & $x$ & 7 & 128 & $x$ & $x$ \\
\hline Peq18 & $162-178(4)$ & $x$ & $x$ & $x$ & $x$ & $x$ & $x$ & $\mathrm{x}$ & $x$ & $x$ & 1 & $x$ & $x$ & $x$ \\
\hline Peq19 & 218-234 (4) & 207,231 & 266,286 & $261,265,277$ & 230 & 257 & 242 & 225,281 & 258,270 & 246 & 6 & $x$ & $x$ & $x$ \\
\hline Peq20 & $214-242(6)$ & $x$ & $\mathrm{x}$ & $x$ & $x$ & 229,245 & 259,267 & $x$ & $x$ & $\mathrm{x}$ & 3 & $x$ & $\mathrm{x}$ & $x$ \\
\hline Peq21 & 168-188 (3) & $248^{\S}$ & 166,180 & 170 & 180 & $x$ & 184 & 146,151 & 180 & 180 & 3 & 180 & 180 & 180 \\
\hline \# Amp & 21 & 13 & 14 & 15 & 11 & 10 & 15 & 15 & 13 & 10 & & 6 & 4 & 4 \\
\hline \# Poly & 21 & 8 & 8 & 11 & 5 & 4 & 12 & 12 & 7 & 4 & & 2 & 1 & 1 \\
\hline
\end{tabular}

*All loci were amplified using the same PCR program and reaction constituents as used with Mauritius parakeet $P$ sittacula eques individuals (see text). $n$, number of individuals tested; $X$, did not amplify; \#Amp, number of loci amplifying per species; \#Poly, number of loci polymorphic per species. ${ }^{8}$, the difference in observed and expected allele sizes suggests an alternative locus may be amplifying to that which was originally cloned and sequenced in the Mauritius parakeet. 


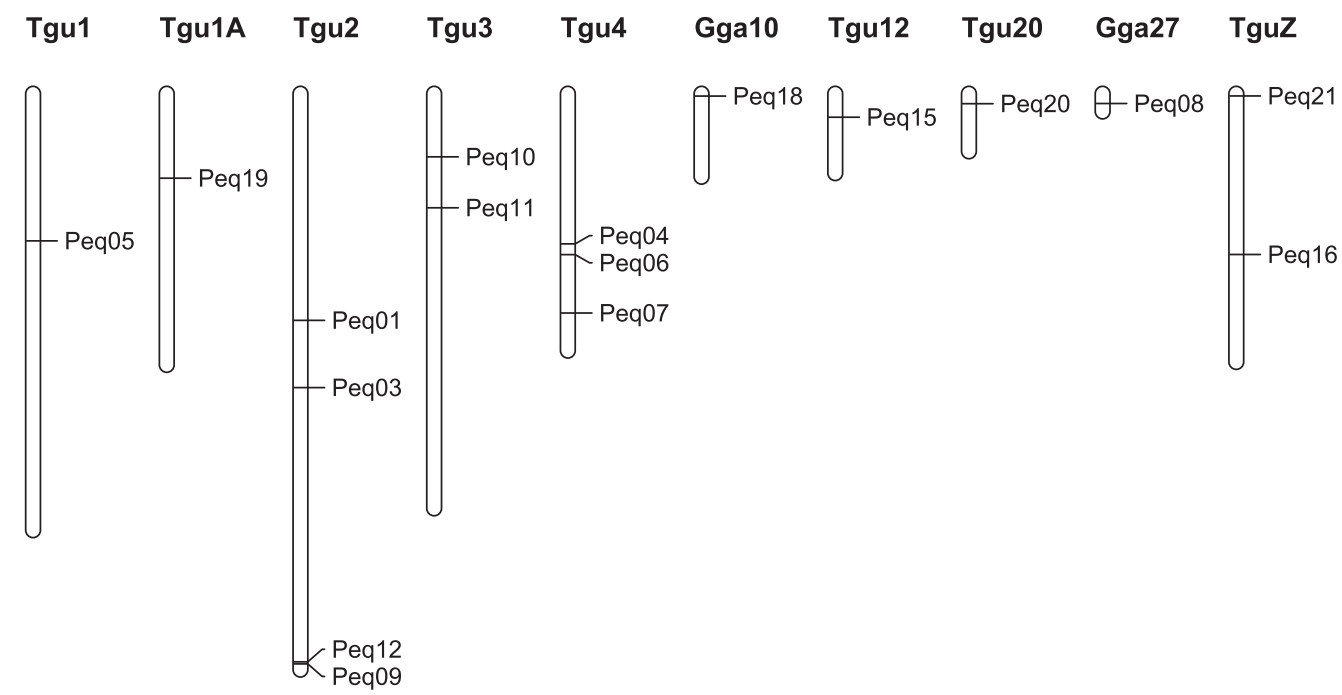

Fig. 1 Chromosome locations in the zebra finch (Taeniopygia guttata) and chicken (Gallus gallus) genome of 15 microsatellite loci characterized in the Mauritius parakeet (Psittacula eques).

using CriMap software (Lander \& Green 1987). Loci were also checked for linkage disequilibrium in unrelated individuals using GenePop version 3.4 and applying a sequential Bonferroni correction (Rice 1989). One pair of loci was assigned to closely neighbouring locations on the same zebra finch chromosome (Peq09 and Peq12, located 0.5 Mb apart on Tgu2; Table 1, Fig. 1) and displayed both linkage (LOD = 10.24) and linkage disequilibrium. The second nearest known neighbouring pair of loci, Peq04 and Peq06 were located $3 \mathrm{Mb}$ apart on Tgu4 and did not display either significant linkage or linkage disequilibrium. Significant linkage was detected between Peq03 and Peq05 (LOD = 4.16) although these loci mapped to different zebra finch chromosomes; these loci were not in linkage disequilibrium. Linkage disequilibrium was additionally indicated between Peq08 and Peq09, which were assigned to different avian chromosomes.

All 21 loci were tested in 13 other species including 10 parrot species (Table 2). Between seven and 21 loci were polymorphic in each of seven parrot species despite testing only one to two individuals. Six loci show particular potential for genetic studies of other parrot species. These six loci were each polymorphic in at least seven other parrot species (Peq01, Peq04, Peq08, Peq11, Peq12 and Peq17).

We have successfully characterized 21 polymorphic microsatellite markers for the Mauritius parakeet. These loci will be used to study the genetic variability of the existent population and the species' mating system.

\section{Acknowledgements}

Hans Cheng, Jon Ekstrom, Winston Kaye, Kate Meares and Tiawanna Taylor kindly provided blood and/or DNA samples. Andy Krupa and Gavin Horsburgh provided technical assistance. We thank Jason Malham and all the Mauritian Wildlife Foundation staff who assisted with the collection of blood samples over several years. This work was supported by the Natural Environment Research Council, UK and Wildlife Vets International, Keighley, UK.

\section{References}

Altschul SF, Madden TL, Schäffer AA et al. (1997) Gapped BLAST and PSI-BLAST: a new generation of protein database search programs. Nucleic Acids Research, 25, 3389-3402.

Dawson DA, Burke T, Hansson B et al. (2006) A predicted microsatellite map of the passerine genome based on chicken-passerine sequence similarity. Molecular Ecology, 15, 1299-1321.

IUCN (2008) Red List of Threatened Species. Available from URL: http://www.iucnredlist.org.

Jones KC, Levine KF, Banks JD (2002) Characterization of 11 polymorphic tetranucleotide microsatellites for forensic applications in California elk (Cervus elaphus canadensis). Molecular Ecology Notes, 2, 435-427.

Lander ES, Green P (1987) Construction of multilocus genetic linkage maps in human. Proceedings of the National Academy of Sciences, USA, 84, 2363-2367.

Malham J, Reuleaux A, Freeman P et al. (2006) Echo Parakeet Management Report, 2006. Mauritian Wildlife Foundation. Vacoas, Mauritius.

Marshall TC, Slate J, Kruuk LEB, Pemberton JM (1998) Statistical confidence for likelihood-based paternity inference in natural populations. Molecular Ecology, 7, 639-655. (http:// helios.bto.edsac.uk/evolgen/cervus/cervus.html).

Nicholls JA, Double MC, Rowell DM, Magrath RD (2000) The evolution of cooperative and pair breeding in thornbills Acanthiza (Pardalotidae). Journal of Avian Biology, 31, 165-176.

Raymond M, Rousset F (1995) GenePop (version 1.2): population genetics software for exact tests and ecumenism. Journal of Heredity, 86, 248-249. (http://genepop.curtin.edu.au/).

Rice WR (1989) Analysing tables of statistical tests. Evolution, 43, 223-225.

doi: 10.1111/j.1755-0998.2009.02621.x

(C) 2009 Blackwell Publishing Ltd 